\title{
Patterns of recurrence after surgery and efficacy of salvage therapy after recurrence in patients with thoracic esophageal squamous cell carcinoma
}

Wenjie $\mathrm{Ni}^{1}$, Jinsong Yang ${ }^{2}$, Wei Deng ${ }^{3}$, Zefen Xiao ${ }^{1 *}$ (D), Zongmei Zhou ${ }^{1}$, Hongxing Zhang ${ }^{1}$, Dongfu Chen ${ }^{1}$, Qinfu Feng ${ }^{1}$, Jun Liang ${ }^{1}$, Jima Lv ${ }^{1}$, Xiaozhen Wang ${ }^{1}$, Xin Wang ${ }^{1}$, Tao Zhang ${ }^{1}$, Nan Bi ${ }^{1}$, Lei Deng ${ }^{1}$ and Wenqing Wang ${ }^{\prime}$

\begin{abstract}
Background: Information on the optimal salvage regimen for recurrent esophageal cancer is scarce. We aimed to assess the patterns of locoregional failure, and evaluate the therapeutic efficacy of salvage therapy along with the prognostic factors in recurrent thoracic esophageal squamous cell carcinoma (TESCC) after radical esophagectomy.

Methods: A total of 193 TESCC patients who were diagnosed with recurrence after radical surgery and received salvage treatment at our hospital were retrospectively reviewed from 2004 to 2014. The patterns of the first failure were assessed. The post-recurrence survival rate was determined using the Kaplan-Meier method and analyzed using the log-rank test. Multivariate prognostic analysis was performed using the Cox proportional hazard model.

Results: The median time of failure was 7.0 months. Among the 193 patients, 163 exhibited isolated locoregional lymph node (LN) recurrence and 30 experienced locoregional $L N$ relapse with hematogenous metastasis. Among the 193 patients, LN recurrence was noted at 302 sites; the most common sites included the supraclavicular (25.8\%; 78/302) and mediastinal LNs (44.4\%; 134/302), particularly stations 1 to 6 for the mediastinal LNs (36.4\%; 110/302). The median overall survival (OS) was 13.1 months after recurrence. In those treated with salvage chemoradiotherapy, with radiotherapy, and without radiotherapy, the 1-year OS rates were 68.5, 55.0, and 28.6\%; the 3-year OS rates were 35.4, 23.8, and 2.9\%; and the 5-year OS rates were 31.8, 17.2, 2.9\%, respectively $(P<0.001)$. Furthermore, patient survival in those who received salvage chemoradiotherapy was significantly better than those treated with salvage radiotherapy alone $(P=0.044)$. Multivariate analysis showed that the pathological TNM stage and salvage treatment regimen were independent prognostic factors.
\end{abstract}

Conclusions: Supraclavicular and mediastinal LN failure were the most common types of recurrence after RO surgery in TESCC patients. Salvage chemoradiotherapy or radiotherapy could significantly improve survival in esophageal cancer with locoregional LN recurrence.

Keywords: Esophageal neoplasm, Recurrence, Salvage treatment, Survival

\footnotetext{
* Correspondence: xiaozefen@sina.com

'Department of Radiation Oncology, National Cancer Center/National Clinical

Research Center for Cancer/Cancer Hospital, Chinese Academy of Medical

Sciences and Peking Union Medical College, No. 17 South Panjiayuan lane,

Chaoyang District, Beijing 100021, China

Full list of author information is available at the end of the article
}

(c) The Author(s). 2020 Open Access This article is distributed under the terms of the Creative Commons Attribution 4.0 International License (http://creativecommons.org/licenses/by/4.0/), which permits unrestricted use, distribution, and reproduction in any medium, provided you give appropriate credit to the original author(s) and the source, provide a link to the Creative Commons license, and indicate if changes were made. The Creative Commons Public Domain Dedication waiver (http://creativecommons.org/publicdomain/zero/1.0/) applies to the data made available in this article, unless otherwise stated. 


\section{Background}

Surgical resection is the mainstay for potentially curable esophageal cancer. However, locoregional recurrence (LRR) is the most common pattern of recurrence, and is noted in up to $23.8-58.0 \%$ of cases, whereas hematogeous metastasis is noted in approximately $5.5-33.0 \%$ of cases [1-6]. The median time to recurrence ranges from 7 to 12.5 months [7-9]. According to the National Comprehensive Cancer Network (NCCN) guidelines, salvage chemoradiotherapy is preferably recommended for such patients with recurrence. However, this recommendation is based on only a few retrospective studies with small sample sizes. Hence, the optimal recommendation for these patients remains unclear, and further studies on salvage treatment are required. In the present study, we aimed to retrospectively analyze the patterns of relapse and therapeutic efficacy of salvage therapy in our institution.

\section{Methods \\ Eligibility criteria}

The study enrolled patients who were diagnosed with LRR and who received salvage treatment at our institution after radical esophagectomy (R0) of TESCC. Patients who underwent neoadjuvant or adjuvant therapy were excluded.

\section{Patients and treatments}

There were 193 patients eligible from January 2004 to December 2014, including 165 male and 28 female patients (median age, 60 years; mean age, $59.7 \pm 8.6$ years). Of these 193 patients, 163 exhibited locoregional LN recurrence alone and 30 exhibited locoregional LN relapse in combination with hematogenous metastases. After recurrence, 48 patients received salvage chemoradiotherapy (SCRT), 109 received salvage radiotherapy (SRT), and 36 received salvage chemotherapy or best supportive care (No RT) due to the presence of LN recurrence in multiple regions and/or hematogenous metastases (Table 1). Radiation therapy was delivered via a $6 \mathrm{MV}$ $\mathrm{X}$-ray linear accelerator. The median dose was $60 \mathrm{~Gy}$ (30 Gy in 1, 40-49 Gy in 6, 50-59 Gy in 45, 60-70 Gy in 103, and unknown in 2 patients). A total of 95 patients received involved field irradiation, whereas 60 patients underwent elective field radiotherapy. The most common chemotherapy regimen included platinum combined with taxane. Of the 66 patients received chemoradiotherapy or chemotherapy, there were 22 cases received paclitaxel plus nedaplatin, 25 received paclitaxel plus cisplatin, 9 received cisplatin plus 5fluorouracil, and 10 received other regimens. Best supportive care involved nutritional support therapy, analgesic therapy, and anti-infective therapy. The main objective was to improve the quality of life.

\section{Follow up}

All patients were assessed at 3-month intervals for the first 2 years after treatment, at 6-month intervals for the next 3 years, and annually thereafter. Computed tomography of the neck, thorax, and upper abdomen using contrast; ultrasonography of the neck and upper abdomen; nuclear bone scanning; conventional blood studies; and biochemistry studies were performed at each followup, in addition to gastric endoscopy, positron emission tomography, or cytologic puncture, as needed. Relapses were classified as LRR and distant metastasis (DM). Overall survival (OS) was defined as the interval between recurrence and death from any cause, loss to follow-up, or last follow-up. The disease-free survival (DFS) time was defined as the interval between surgery and first recurrence.

\section{Statistical analysis}

The survival rate was calculated by the Kaplan-Meier method and analyzed using the log-rank test. Multivariate analyses were performed to identify the prognostic factors for post-recurrence OS using the Cox proportional hazards model. All tests were two-sided, and $P$ values $<0.05$ were considered to indicate statistical significance. All the statistical analyses were performed using the Statistical Package for Social Sciences 23.0 software (SPSS Inc., Chicago, IL).

\section{Results \\ Distribution of resected LNs and failure patterns in locoregional LNs}

A total of 148 patients received Sweet esophagectomy and 45 patients underwent the Ivor-Lewis approach. The upper mediastinal LNs were defined as those in the tracheoesophageal groove; with the recurrent laryngeal nerve; at stations $2,3 \mathrm{p}, 4$, and 5; and with the paraaorta. In total, $201 \mathrm{LNs}$ were resected, including 34 that were confirmed as having metastases (16.9\%). Furthermore, 2086 LNs were resected from the paraesophageal and subcarina regions, and only $133(6.4 \%)$ had metastases. The metastasis rate of abdominal LNs was 7.2\% (131/1812; Fig. 1a).

Supraclavicular and mediastinal LNs were the most common sites of recurrence (16.6 and 46.6\%, respectively). The recurrence rate in the upper abdominal LNs was only $8.8 \%$. Among the 193 patients, 302 sites with LRR were noted, including $36.4 \%(110 / 302)$ of sites in the 1 to 6 mediastinal regional LNs and 25.8\% (78/302) of sites in the supraclavicular LNs (Table 2 and Fig. 1b).

\section{Survival rates in different subgroups}

The final follow-up was performed on November 24, 2018 , with a median follow-up of 96.8 months. The median survival time after recurrence was 13.1 months. The 
Table 1 Patient demographic and clinical characteristics

\begin{tabular}{|c|c|c|c|c|c|}
\hline & Number 193(\%) & SCRT 48(\%) & SRT 119(\%) & No RT 36(\%) & $p$ \\
\hline Sex & & & & & 0.139 \\
\hline Male & $165(85.5)$ & $45(93.8)$ & $89(81.7)$ & $31(86.1)$ & \\
\hline Female & $28(14.5)$ & $3(6.3)$ & $20(18.3)$ & $5(13.9)$ & \\
\hline Age, years & & & & & 0.054 \\
\hline$\leq 60$ & $100(51.8)$ & $32(66.7)$ & $50(45.9)$ & $18(50.0)$ & \\
\hline$>60$ & $93(48.2)$ & $16(33.3)$ & $59(54.1)$ & $18(50.0)$ & \\
\hline Location of the primary tumor & & & & & 0.645 \\
\hline Upper & $27(14.0)$ & $8(16.7)$ & $14(12.8)$ & $5(13.9)$ & \\
\hline Middle & $91(47.2)$ & $25(52.1)$ & $52(47.7)$ & $14(38.9)$ & \\
\hline Lower & 75 (38.9) & $15(31.3)$ & $43(39.4)$ & $17(47.2)$ & \\
\hline Surgery method & & & & & $<0.001$ \\
\hline Sweet approach & $148(76.7)$ & $10(37.0)$ & 67 (73.6) & $71(94.7)$ & \\
\hline Ivor-Lewis approach & $45(23.3)$ & $17(63 . .0)$ & $24(26.4)$ & $4(5.3)$ & \\
\hline Differentiation degree & & & & & 0.488 \\
\hline Well & $25(13.0)$ & $6(12.5)$ & $15(13.8)$ & $4(11.1)$ & \\
\hline Median & $113(58.5)$ & $26(54.2)$ & $66(60.6)$ & $21(58.3)$ & \\
\hline Poor & $54(28.0)$ & $16(33.3)$ & $28(25.7)$ & $10(27.8)$ & \\
\hline Unknown & $1(0.5)$ & - & - & - & \\
\hline Pathological T stage* & & & & & 0.097 \\
\hline $\mathrm{T} 1$ & $5(2.6)$ & $1(2.1)$ & $2(1.8)$ & $2(5.6)$ & \\
\hline $\mathrm{T} 2$ & $35(18.1)$ & $10(20.8)$ & $20(18.3)$ & $5(13.9)$ & \\
\hline T3 & $149(77.2)$ & $37(77.1)$ & $86(78.9)$ & $26(72.2)$ & \\
\hline T4a & $4(2.1)$ & $0(0.0)$ & $1(0.9)$ & $2(8.3)$ & \\
\hline Pathological N stage* & & & & & $<0.001$ \\
\hline No & $106(54.9)$ & 35 (72.9) & $64(58.7)$ & $7(19.4)$ & \\
\hline $\mathrm{N} 1$ & $43(22.3)$ & $5(10.4)$ & $24(22.0)$ & $14(38.9)$ & \\
\hline N2 & $30(15.5)$ & $4(8.3)$ & $16(14.7)$ & $10(27.8)$ & \\
\hline N3 & $14(7.3)$ & $4(8.3)$ & $5(4.6)$ & $5(13.9)$ & \\
\hline Pathological TNM stage* & & & & & 0.001 \\
\hline IB & $26(13.5)$ & $7(14.6)$ & $17(15.6)$ & $2(5.6)$ & \\
\hline$\| \mathrm{A}$ & $80(41.5)$ & $28(58.3)$ & $47(43.1)$ & $5(13.9)$ & \\
\hline$\| B$ & $10(5.2)$ & $2(4.2)$ & $4(3.7)$ & $4(11.1)$ & \\
\hline$\| \mathrm{A}$ & $36(18.7)$ & $4(8.3)$ & $21(19.3)$ & $11(30.6)$ & \\
\hline IIIB & $25(13.0)$ & $3(6.3)$ & 15 (13.8) & $7(19.4)$ & \\
\hline$\| I I C$ & $16(8.3)$ & $4(8.3)$ & $5(4.6)$ & $7(19.4)$ & \\
\hline Radiation dose & & & & & 0.030 \\
\hline$<60 G y$ & $52(33.5)$ & $22(45.8)$ & $30(28.0)$ & - & \\
\hline$\geq 60 \mathrm{~Gy}$ & $103(66.5)$ & $26(54.2)$ & 77 (72.0) & - & \\
\hline
\end{tabular}

* 7th UICC

1-, 3-, and 5-year OS rates after recurrence were 53.5, 22.7 , and $17.9 \%$, respectively.

The median survival time for patients with SCRT, SRT, and No RT were 23.2, 16.2, and 5.7 months, respectively. Moreover, in those with SCRT, SRT, and No RT, the 1year OS rates were 68.5, 55.0, and 28.6\%; 3-year OS rates were 35.4, 23.8, and 2.9\%; and 5-year OS rates were 31.8, 17.2 , and $0.0 \%$, respectively $(P<0.001$; Fig. 2$)$. Moreover, there was a significant survival benefit in favor of the SCRT group, relative to the SRT group $(P=0.044)$.

Two patients were excluded because the radiation dose was unknown. A total of 52 patients received radiation 

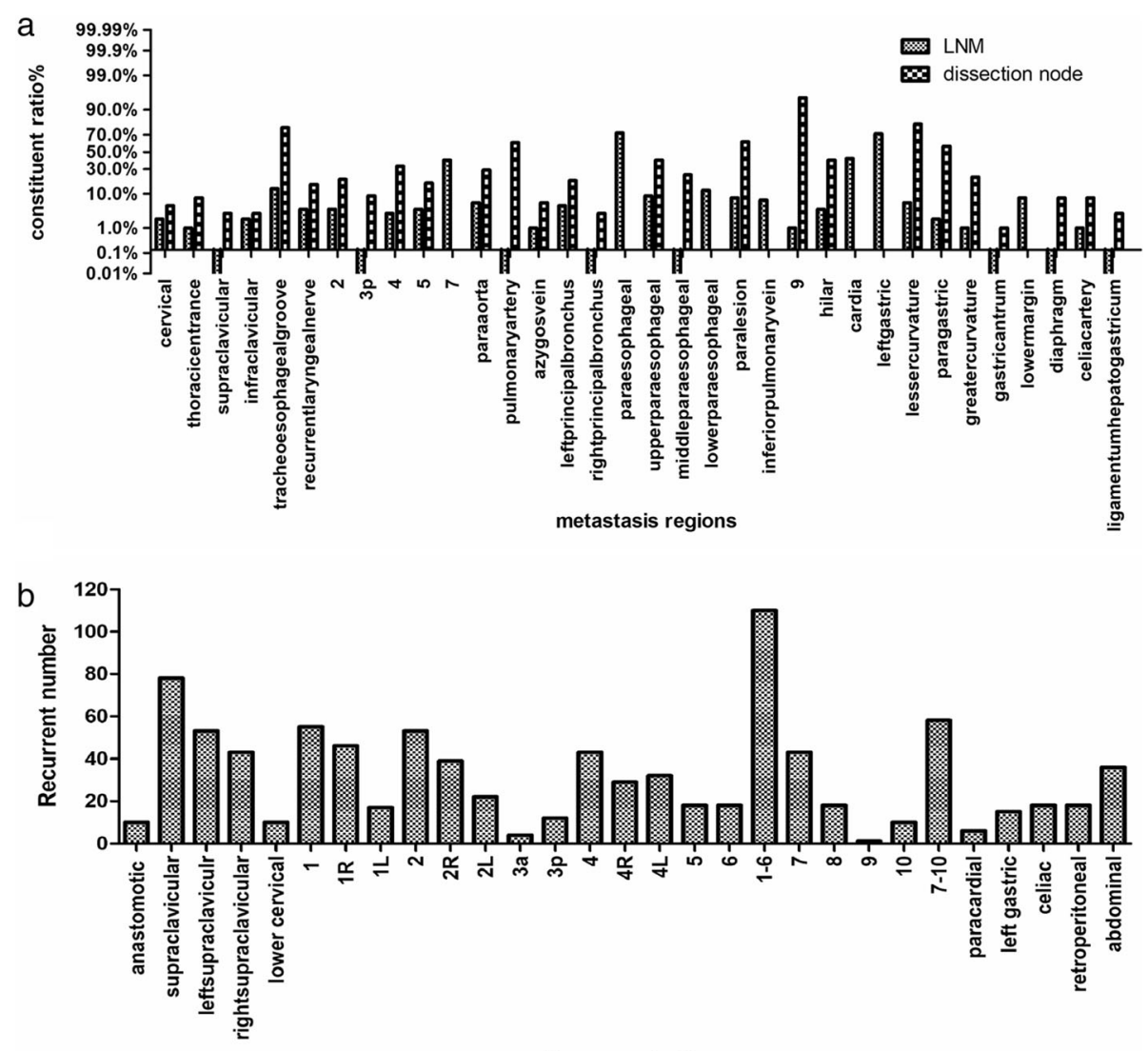

Recurrent site

Fig. 1 a Distribution of lymph node resection (LNM: lymph node metastases); $\mathbf{b}$ Distribution of recurrent lymph nodes

at a dose $<60 \mathrm{~Gy}$, and their median survival time after salvage treatment was 12.0 months; the 1-, 3-, and 5-year OS rates of these patients were $48.5,20.3$, and $13.9 \%$, respectively. Moreover, 103 patients received a dose of $\geq 60 \mathrm{~Gy}$, and their median survival time was 21.9 months; the 1-, 3-, and 5-year OS rates in these patients were $65.5,31.2$, and $25.3 \%$, respectively. The differences in OS rates between these groups were significant $(P=0.026$; Fig. 3).

LN recurrence above the diaphragm was noted in 139 cases, recurrence in the subphrenic LNs was noted in 24

Table 2 Patterns of recurrence in the locoregional lymph nodes

\begin{tabular}{lll}
\hline Site of lymph node recurrence & Number & $\%$ \\
\hline Supraclavicular LN & 32 & 16.6 \\
Mediastinal LN & 90 & 46.6 \\
Upper abdominal LN & 17 & 8.8 \\
Supraclavicular and mediastinal LN & 37 & 19.2 \\
Supraclavicular and upper abdominal LN & 3 & 1.6 \\
Mediastinal and abdominal LN & 11 & 5.7 \\
Supraclavicular, mediastinal, and upper abdominal LN & 3 & 1.6 \\
\hline
\end{tabular}

cases, and LN recurrence with hematogenous metastasis was observed in 30 cases; in these 3 scenarios, the 1-year OS rates were $57.5,34.8$, and $49.8 \%$; 3 -year OS rates were 28.5, 4.3, and 10.7\%; and 5-year OS rates were 22.0, 4.3 , and $10.7 \%$, respectively ( $P=0.001$; Fig. $4 a)$. Single region recurrence (SRR) was defined as $\mathrm{LN}$ recurrence in only one region, whereas multiple region recurrence

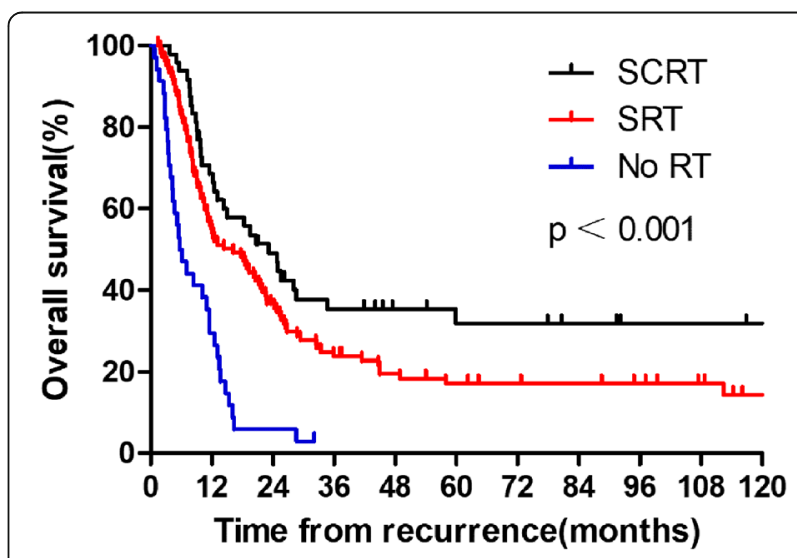

Fig. 2 The overall survival rates after recurrence according to different salvage regimens 


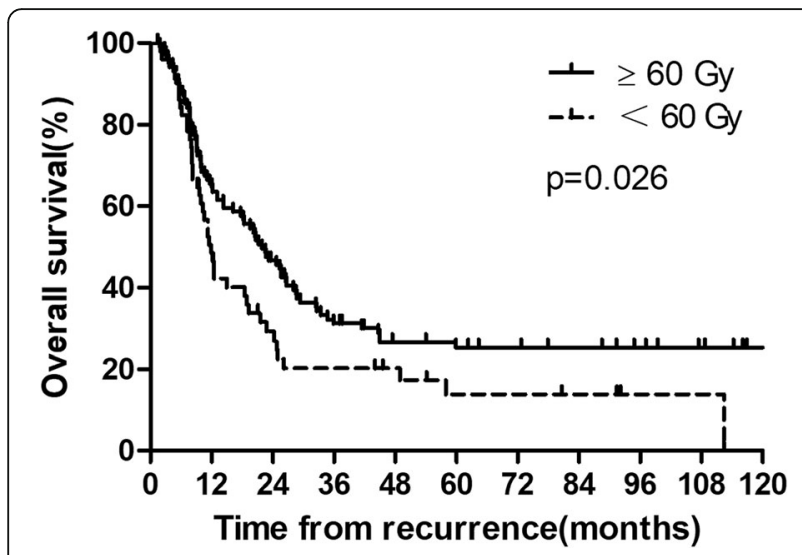

Fig. 3 The overall survival rates after recurrence, stratified by the dose of radiation

(MRR) was defined as relapse in more than $1 \mathrm{LN}$ region. In total, $83(43.0 \%)$ and $110(57.0 \%)$ patients exhibited SRR and MRR, respectively, and the corresponding 1-, 3 -, and 5-year OS rates were $54.8,31.1$, and $29.4 \%$ for the SRR and 52.6, 15.9, and 9.2\% for the MRR cases, respectively $(P=0.041$; Fig. $4 \mathrm{~b})$.

Univariate analysis indicated that the pathological $\mathrm{N}$ stage, pathological TNM stage, salvage treatment regimen, recurrence site, and number of recurrent regions were associated with the OS. Furthermore, multivariate analysis showed that the pathological TNM stage and salvage treatment regimen were independent prognostic factors (Table 3).

\section{Discussion}

Esophagectomy remains the standard treatment for esophageal cancer. The NCCN guidelines recommend surveillance after radical resection, regardless of the status of primary tumor or regional LN. However, the recurrence rate in these cases remains high, which is a major reason for failure after surgery.

Recent studies have found that supraclavicular and mediastinal LN recurrence are the most common [5,
10-12], although the abdominal LN relapse rate remains at approximately $9.3 \%[11,12]$, consistent with our study. LN dissection around the tracheoesophageal groove and recurrent laryngeal nerve is known to be difficult via Sweet esophagectomy. In the present study, only $23.3 \%$ of patients underwent Ivor-Lewis esophagectomy, which reportedly has a higher LN retrieval relative to the Sweet approach, particularly for upper mediastinal LNs [13]. In contrast, the paraesophageal, subcarina, and abdominal LNs are easy to resect via both surgical procedures. Therefore, the bilateral supraclavicular (16.6\%) and upper mediastinal $(36.4 \%)$ regions were the main sites of LN recurrence in our study.

The possible treatments for recurrence include SCRT, surgical resection, SRT, chemotherapy, or best supportive care. The NCCN guidelines recommend SCRT as preferable in patients with LRR who do not receive prior radiotherapy. However, there is a lack of studies with a large sample size on the most effective salvage therapeutic strategies.

The survival rate of salvage treatment is reportedly poor after recurrence. The 1- and 3-year OS rates are approximately $45.9-47.1 \%$ and $10.6-17.1 \%$, respectively, whereas the 5 -year OS rates are only $4.3-6.4 \%[14,15]$. In the present study, the 1-, 3-, and 5-year OS rates were 53.5, 22.7 , and $17.9 \%$ respectively, which are slightly higher than the above-mentioned results. There may be some key reasons for this difference. First, early recurrence detection was the main focus for improving the salvage treatment effect in our study, so that patients could receive prompt treatment. The median time to recurrence was 7.0 months in our study, compared to $13.0-15.0$ months in the abovementioned studies. Second, 83 (43.0\%) patients had SRR in our study, and the OS of these cases was markedly higher than that of cases with MRR. Moreover, the present study included approximately $55.0 \%$ of patients with original pathological stage IB-IIA, which was more than that (36.3\%) in the study of Su et al. [15]. Finally, 81.3\% (157/193) patients received SCRT and SRT in our research, whereas only $42.6 \%$ (81/190) patients received SRT in the study of Su et al. [15]. Therefore, close follow-
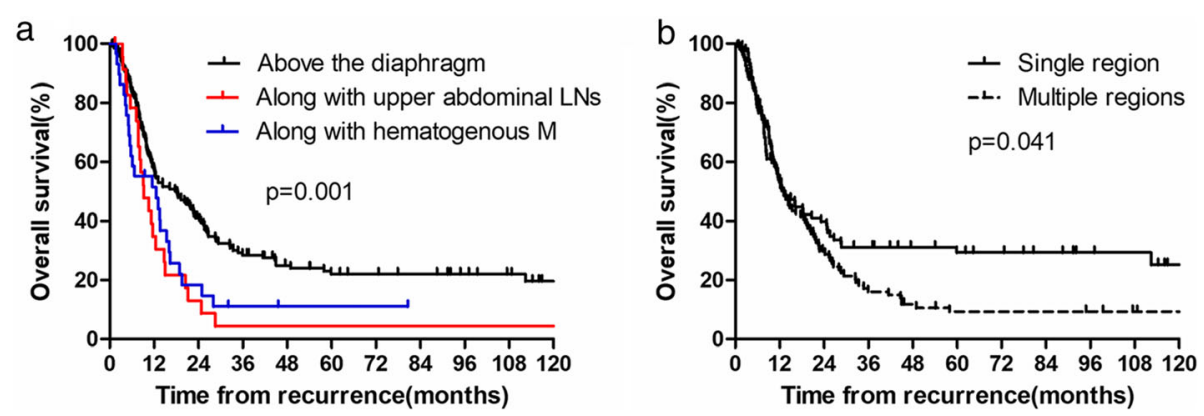

Fig. 4 a The overall survival rates after recurrence according to the site of recurrence in relation to the diaphragm; $\mathbf{b}$ The overall survival rates after recurrence based on the number of recurrent $L N$ regions 
Table 3 Univariate and multivariate analysis for overall survival after recurrence

\begin{tabular}{|c|c|c|c|c|c|}
\hline \multirow[t]{2}{*}{ Factors } & \multirow[t]{2}{*}{ Number } & \multicolumn{2}{|l|}{ Univariate } & \multicolumn{2}{|l|}{ Multivariate } \\
\hline & & $\mathrm{HR}(95 \% \mathrm{Cl})$ & $P$ & $\mathrm{HR}(95 \% \mathrm{Cl})$ & $P$ \\
\hline Pathological N stage & & & $<0.001$ & & $<0.001$ \\
\hline pNO & 106 & 1 & & 1 & \\
\hline $\mathrm{pN}+$ & 87 & $1.984(1.438-2.737)$ & & $0.507(0.360-0.714)$ & \\
\hline Pathological TNM stage & & & $<0.001$ & - & - \\
\hline$|/| \mid$ & 116 & 1 & & & \\
\hline III & 77 & $2.353(1.697-3.263)$ & & & \\
\hline Salvage treatment & & & $<0.001$ & & $<0.001$ \\
\hline SCRT & 48 & 1 & & 1 & \\
\hline SRT & 109 & $1.510(1.002-2.276)$ & 0.049 & $1.419(0.940-2.142)$ & 0.096 \\
\hline No RT & 36 & $4.175(2.529-6.891)$ & $<0.001$ & $3.246(1.931-5.458)$ & $<0.001$ \\
\hline Site of recurrence & & & 0.002 & - & - \\
\hline Above the diaphragm & 139 & 1 & & & \\
\hline Along with upper abdominal LN recurrence & 24 & $1.967(1.234-3.136)$ & 0.004 & & \\
\hline Along with hematogenous metastases & 30 & $1.816(1.177-2.804)$ & 0.007 & & \\
\hline Number of LN recurrence regions & & & 0.042 & - & - \\
\hline Single region & 83 & 1 & & & \\
\hline Multiple regions & 110 & $1.408(1.012-1.958)$ & & & \\
\hline
\end{tabular}

up, early recurrence detection, SRR, and timely salvage treatment were vital factors leading to favorable results in the recurrent patients.

The optimal salvage strategies in patients with LRR remain controversial. SCRT was found to confer better survival than SRT in the present study, consistent with that noted in previous studies [16, 17]. Although these studies were retrospective in nature, they support the importance of salvage treatment for LRR, particularly that of SCRT. However, the outcomes of SCRT and SRT after recurrence remain unclear. Nemoto et al. [9] found that SRT combined with chemotherapy had better local control rates but not OS. In contrast, SRT was found to be superior to SCRT in another small sample size study of patients with LRR and DM [18]. In the present study, SCRT had better OS than SRT, and the difference was significant. The possible reason to explain this difference includes the using of three-dimensional conformal radiotherapy in our institution besides above-mentioned early recurrence detection and earlier pathological stage. It has been reported that three-dimensional conformal radiotherapy can increase the local control rate and OS rate in nasopharyngeal carcinoma [19] and lung cancer [20]. Furthermore, chemotherapy with radiotherapy could increase the radiosensitivity [21] and improve OS, relative to radiotherapy alone, in nasopharyngeal carcinoma [22] and esophageal cancer [23]. Thus, SCRT appears to be a superior option for specific patients.

Furthermore, the specific salvage radiation dose differs among various studies. Shioyama et al. [24] assessed 66 patients receiving a dose of $\geq 50 \mathrm{~Gy}$ and 16 patients receiving a dose of <50 Gy, and observed 2-year and 5year OS rates of $26.0,13.0$, and $10.0 \%, 0.0 \%$, respectively $(P=0.003)$; accordingly, the researchers believed that 50 Gy was the best radiation dose for salvage treatment. Zhang and colleagues [25] found that an irradiation dose of $\geq 60$ Gy could improve the OS among patients with recurrent esophageal cancer after surgery $(P<0.05)$. Another retrospective study with a small sample size was not able to determine a significant radiation dose [18]. In the present study, the 5-year OS was $25.3 \%$ in the $\geq 60$ Gy group, as compared to $13.9 \%$ in the <60 Gy group $(P=0.026)$. Thus, we believe that a salvage dose of at least $60 \mathrm{~Gy}$ might be more reasonable and effective for LRR. However, as our study is retrospective in nature and was performed at a single center, it might suffer from and heterogeneity; hence, a prospective trial may be needed to confirm these findings.

Cases with LN recurrence above the diaphragm exhibited better OS than those with recurrence at the subphrenic LNs and with hematogenous metastasis. Some studies found that the location of regional LN metastases influenced survival, particularly that of celiac LN metastasis $[26,27]$. However, research related to abdominal LN recurrence remains insufficient. A retrospective study showed that although the location of LN metastasis did not significantly influence survival, patients with LN metastasis around the abdominal aorta had poor survival [28]. There may be some reasons for this finding. First, LN recurrence under the diaphragm often presents with 


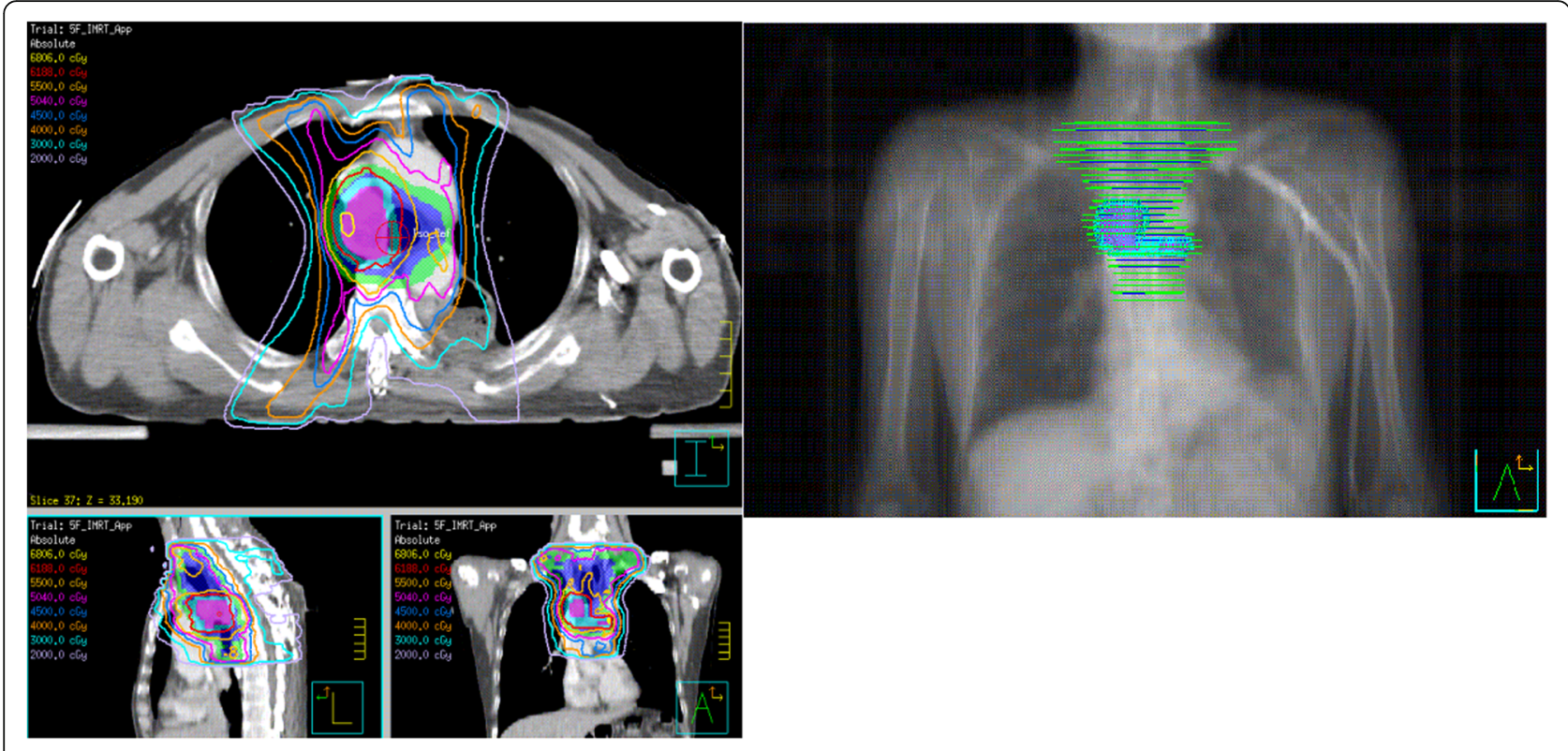

Fig. 5 Target contouring and planning design for elective field radiotherapy after recurrence. GTVnd (pink area), CTV (blue area), PGTVnd (sky blue area), PTV (green area). The prescribed dose (SIB-IMRT) for 95\% PTV was 50.4 Gy/1.8 Gy/28 f, and for 95\% PGTVnd was $61.88 \mathrm{~Gy} / 2.21 \mathrm{~Gy} / 28 \mathrm{f}$. The concurrent chemotherapy regimen included liposomal paclitaxel $\left(135 \mathrm{mg} / \mathrm{m}^{2} \mathrm{IV}\right.$ ) on day 1 and nedaplatin $\left(50 \mathrm{mg} / \mathrm{m}^{2} \mathrm{IV}\right)$ on day 1 , which were cycled every 21 days. A total of 2 cycles were delivered
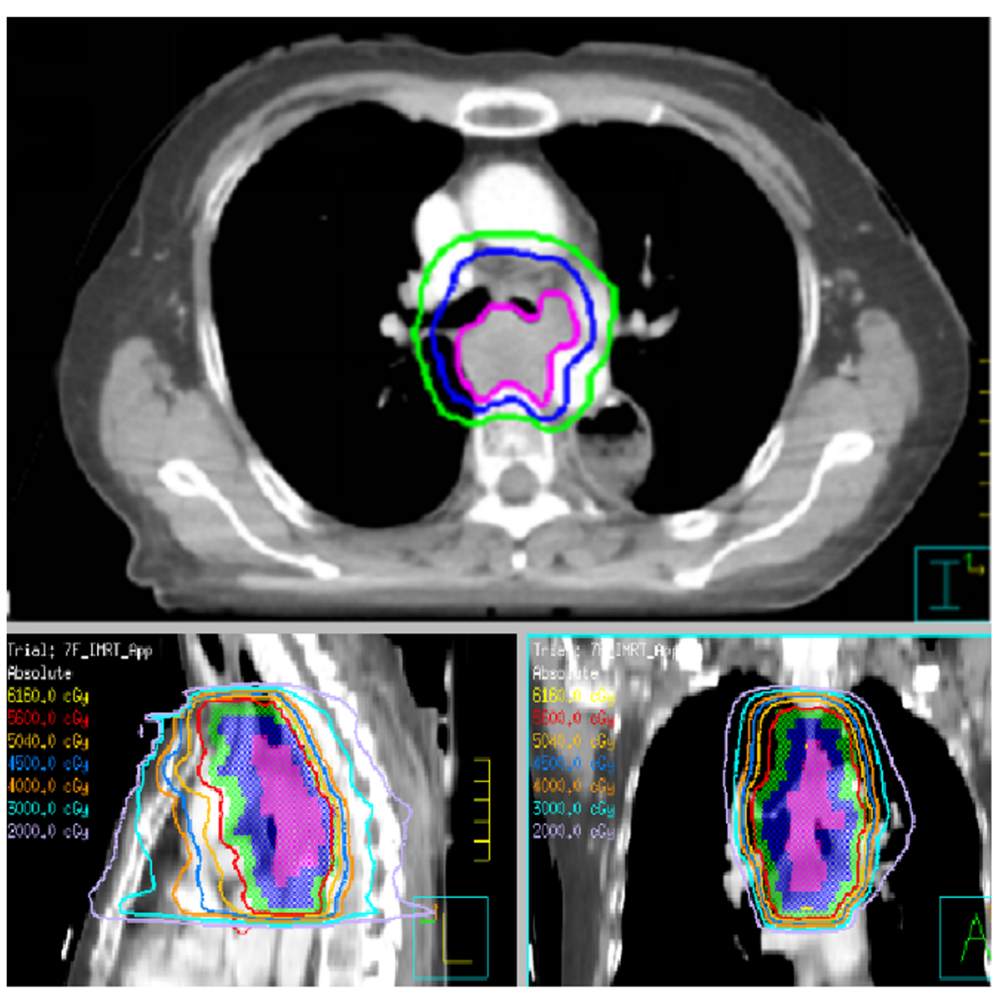

Fig. 6 Target contouring and planning design for involved field irradiation. GTVnd (pink area), CTV (blue area), PTV (green area). The prescribed dose (IMRT) for 95\% PTV was 56 Gy/2 Gy/28 f 
necrosis and hypoxia, which are associated with poor radiosensitivity. Second, the prescription dose in these cases is insufficient due to limitations of the stomach and intestine. Third, patients with abdominal LN recurrence might be prone to developing hematogenous metastasis. Nevertheless, further research is needed to determine the reason of poor OS for these patients.

In addition, we found that the prognosis of patients with MRR was significantly poorer than that of patients with SRR. Similarly, some studies found that the number of recurrent LNs and number of recurrent LN regions were prognostic factors [2, 7, 28-31]. Researchers considered that patients with multiple recurrent tumors might be considered as having systemic recurrence at the time of diagnosis. This would affect the proportion of patients who receive radiation therapy due to the heavy tumor burden, large target area, and poor status.

Thus, the clinical findings of the present study suggest that salvage concurrent chemoradiotherapy could not only improve the synergistic effect of radiotherapy, but can also control the potential metastasis of subclinical lesions. The field and dose of radiation should be determined based on the specific recurrence type. Simultaneous Integrated Boost Intensity-Modulated Radiation Therapy (SIB-IMRT) was the optimal irradiation technology to increase the dose of regions at high risk, while simultaneously reducing the dose to organs at risk (OAR; Fig. 5). However, recurrence at anastomotic sites, near anastomotic sites, and near the remnant stomach can be treated with sequential radiotherapy to avoid stenosis of the anastomotic stoma or complications of the remnant stomach. For patients with heavy burden of local recurrence or extensive metastasis at multiple sites who cannot undergo radical treatment, palliative therapy was used to improve the quality of life (Fig. 6).

Multivariate analysis indicated that only the original pathological TNM stage and salvage treatment regimen were independent prognostic factors. Therefore, the salvage treatment regimen is vital in patients with recurrence after radical surgery. We suggest that SCRT or SRT with a dose of $\geq 60$ Gy is the optimal choice for LRR.

The retrospective nature of the present study is a limitation. Moreover, the number of patients across the 3 groups was not balanced. Hence, these findings should be confirmed in a large-scale prospective study.

\section{Conclusions}

Failure was most common in the supraclavicular and upper mediastinal LNs after R0 surgery in TESCC patients. SCRT or SRT with a dose of at least $60 \mathrm{~Gy}$ could significantly improve $\mathrm{OS}$ in esophageal cancer with locoregional LN recurrence.

\section{Abbreviations}

Cl: Confidence intervals; CTV: Clinical target volume; DFS: Disease-free survival; DM: Distant metastasis; GTVnd: Metastatic regional nodes; HR: Hazard ratios; IMRT: Intensity-Modulated Radiation Therapy; IV: Intravenous intravenously; LN: Lymph node; LNM: Lymph node metastases; LRR: Locoregional recurrence; MRR: Multiple region recurrence; NCCN: National Comprehensive Cancer Network; OAR: Organs at risk; OS: Overall survival; PGTVnd: Planning metastatic regional nodes; PTV: Planning target volume; SCRT: Salvage chemoradiotherapy; SIBIMRT: Simultaneous Integrated Boost Intensity-Modulated Radiation Therapy; SRR: Single region recurrence; SRT: Salvage radiotherapy; TESCC: Thoracic esophageal squamous cell carcinoma; UICC: Union for international cancer control

\section{Acknowledgements}

We thank all the patients who participated in this trial, all participating investigators who devote their time and passion in the implementation of this study.

\section{Authors' contributions}

ZFX made substantial contributions to the conception and design of the study, revised the article critically for important intellectual content and gave final approval of the version to be published; WJN collected research data and drafted the manuscript; JSY and WD participated in designing and conducting the study; ZMZ, HXZ, DFC, QFF, JL, JML, XZW, XW, TZ, NB, LD, WQW provided patients; All authors read and approved the final manuscript.

\section{Funding}

This work was supported by the Capital Fund for Health Improvement and Research [grant number 2016-2-4021] and Beijing Hope Run Special Fund of Cancer Foundation of China [grant number LC2016L04]. The funding source has no role in study design, data collection, analysis, interpretation, the writing of the manuscript, or the decision to submit the current study.

\section{Availability of data and materials}

All data generated or analysed during this study are included in this published article.

\section{Ethics approval and consent to participate}

Institutional review board approval was obtained for the retrospective study from the ethical committee of the Chinese Academy of Medical Sciences. Written informed consent is obtained from all participants.

\section{Consent for publication}

Not applicable.

\section{Competing interests}

The authors declare that they have no competing interests.

\section{Author details}

1Department of Radiation Oncology, National Cancer Center/National Clinical Research Center for Cancer/Cancer Hospital, Chinese Academy of Medical Sciences and Peking Union Medical College, No. 17 South Panjiayuan lane, Chaoyang District, Beijing 100021, China. ${ }^{2}$ Cancer Center, Union Hospital, Tongji Medical College, Huazhong University of Science and Technology, Wuhan, China. ${ }^{3}$ Key Laboratory of Carcinogenesis and Translational Research (Ministry of Education/Beijing), Department of Radiation Oncology, Peking University Cancer Hospital \& Institute, Beijing, China.

Received: 30 August 2019 Accepted: 11 February 2020

Published online: 22 February 2020

\section{References}

1. Hsu PK, Wang BY, Huang CS, Wu YC, Hsu WH. Prognostic factors for postrecurrence survival in esophageal squamous cell carcinoma patients with recurrence after resection. J Gastrointest Surg. 2011;15:558-65.

2. Miyata H, Yamasaki M, Kurokawa Y, Takiguchi S, Nakajima K, Fujiwara Y, Konishi K, Mori M, Doki Y. Survival factors in patients with recurrence after curative resection of esophageal squamous cell carcinomas. Ann Surg Oncol. 2011;18:3353-61. 
3. Lu J, Tao H, Song D, Chen C. Recurrence risk model for esophageal cancer after radical surgery. Chin J Cancer Res. 2013;25:549-55.

4. Oppedijk V, van der Gaast A, van Lanschot JJB, van Hagen P, van Os R, van Rij CM, et al. Patterns of recurrence after surgery alone versus preoperative Chemoradiotherapy and surgery in the CROSS trials. J Clin Oncol. 2014;32: 385-91.

5. Liu Q, Cai XW, Wu B, Zhu ZF, Chen HQ, Fu XL. Patterns of failure after radical surgery among patients with thoracic esophageal squamous cell carcinoma: implications for the clinical target volume design of postoperative radiotherapy. PLoS One. 2014;9:e97225.

6. Guo XF, Mao T, Gu ZT, Ji CY, Fang WT, Chen WH. Clinical study on postoperative recurrence in patients with pNO esophageal squamous cell carcinoma. J Cardiothorac Surg. 2014;9:150.

7. Kawamoto T, Nihei K, Sasai K, Karasawa K. Clinical outcomes and prognostic factors of chemoradiotherapy for postoperative lymph node recurrence of esophageal cancer. Jpn J Clin Oncol. 2018;48:259-64.

8. Zhou Y, Ding N, Wang L, Liu W, Jiang M, Lu J. Salvage radiochemotherapy for lymph node recurrence after radical surgery of esophageal cancer. Medicine. 2018:97:e9777.

9. Nemoto K, Ariga H, Kakuto Y, Matsushita H, Takeda K. Radiation therapy for loco-regionally recurrent esophageal cancer after surgery. Radiother Oncol. 2001;2:165-8

10. Cai W, Xin P. Pattern of relapse in surgical treated patients with thoracic esophageal squamous cell carcinoma and its possible impact on target delineation for postoperative radiotherapy. Radiother Oncol. 2010;96:104-7.

11. Liu J, Cai X, Liu Q, Li H, Cheng Y, Fu X. Characteristics of the local recurrence pattern after curative resection and values in target region delineation in postoperative radiotherapy for lower thoracic esophageal squamous cell cancer. Thorac Cancer. 2017;8:630-3.

12. Chen J, Cai W, Lin Y, Chen Y, Zheng Q, Pan J, Chen C. Patterns and rates of abdominal lymphatic metastasis following esophageal carcinoma. PLoS One. 2017;12:e185424.

13. Li B, Xiang J, Zhang Y, Li H, Zhang J, Sun Y, et al. Comparison of Ivor-Lewis vs sweet Esophagectomy for esophageal squamous cell carcinoma. JAMA Surg. 2015;150:292.

14. Raoul JL, Le Prise E, Meunier B, Julienne V, Etienne PL, Gosselin M, Launois B. Combined radiochemotherapy for postoperative recurrence of oesophageal cancer. Gut. 1995;37:174-6.

15. Su $X$, Zhang $D$, Zhang $X$, Lin $P$, Long $H$, Rong $T$. Prognostic factors in patients with recurrence after complete resection of esophageal squamous cell carcinoma. J Thorac Dis. 2014;6:949-57.

16. Sang M, Chen J, Liu H. Result of treatment for recurrence of esophageal cancer after surgery. China Oncol. 2000;10:35-7.

17. Lu JC, Kong C, Tao H. Radiotherapy with or without concurrent chemotherapy for lymph node recurrence after radical surgery of thoracic esophageal squamous cell carcinoma. Int J Radiat Oncol Biol Phys. 2010;78:710-4.

18. Li B, Zhang W, Zhao L, Liu N, Pang Q, Yuan Z, et al. Therapeutic strategies for recurrence of esophageal cancer after radical resection. Chin J Clin Oncol. 2013:40:1553-7.

19. Lai SZ, Li WF, Chen L, Luo W, Chen YY, Liu LZ, et al. How does intensitymodulated radiotherapy versus conventional two-dimensional radiotherapy influence the treatment results in nasopharyngeal carcinoma patients? Int J Radiat Oncol Biol Phys. 2011;80:661-8.

20. Sher DJ, Koshy M, Liptay MJ, Fidler MJ. Influence of conformal radiotherapy technique on survival after chemoradiotherapy for patients with stage II non-small cell lung cancer in the National Cancer Data Base. Cancer Am Cancer Soc. 2014;120:2060-8.

21. Steel GG, Peckham MJ. Exploitable mechanisms in combined radiotherapychemotherapy: the concept of additivity. Int J Radiat Oncol Biol Phys. 1979; 5:85-91.

22. Al-Sarraf M, LeBlanc M, Giri PG, Fu KK, Cooper J, Vuong T, et al. Chemoradiotherapy versus radiotherapy in patients with advanced nasopharyngeal cancer: phase III randomized intergroup study 0099. J Clin Oncol. 1998:16:1310-7.

23. Cooper JS, Guo MD, Herskovic A, Macdonald JS, Martenson JJ, Al-Sarraf M, et al. Chemoradiotherapy of locally advanced esophageal cancer: long-term follow-up of a prospective randomized trial (RTOG 85-01). Radiat Ther Oncol Group JAMA. 1999;281:1623-7.

24. Shioyama Y, Nakamura K, Ohga S, Nomoto S, Sasaki T, Yamaguchi T, et al. Radiation therapy for recurrent esophageal Cancer after surgery: clinical results and prognostic factors. Jpn J Clin Oncol. 2007;37:918-23.
25. Zhang J, Peng F, Li N, Liu Y, Xu Y, Zhou L, et al. Salvage concurrent radiochemotherapy for post-operative local recurrence of squamous-cell esophageal cancer. Radiat Oncol. 2012;7:93.

26. Anderegg MC, Lagarde SM, Jagadesham VP, Gisbertz SS, Immanuel A, Meijer $S \mathrm{~L}$, et al. Prognostic significance of the location of lymph node metastases in patients with adenocarcinoma of the distal esophagus or Gastroesophageal junction. Ann Surg. 2016;264:847-53.

27. Okamura A, Watanabe M, Kozuki R, Toihata T, Yuda M, Imamura Y, Mine S. Supraclavicular and celiac metastases in squamous cell carcinoma of the middle thoracic esophagus. Langenbeck's Arch Surg. 2018;403:977-84.

28. Nakamura T, Ota M, Narumiya K, Sato T, Ohki T, Yamamoto M, Mitsuhashi N. Multimodal treatment for lymph node recurrence of esophageal carcinoma after curative resection. Ann Surg Oncol. 2008;15:2451-7.

29. Jingu K, Matsushita H, Takeda K, Umezawa R, Takahashi C, Sugawara T, et al. Long-term results of radiotherapy combined with nedaplatin and 5 fluorouracil for postoperative loco-regional recurrent esophageal cancer: update on a phase II study. BMC Cancer. 2012;12:542.

30. Ma DY, Tan BX, Liu M, Li XF, Zhou YQ, Lu Y. Concurrent three-dimensional conformal radiotherapy and chemotherapy for postoperative recurrence of mediastinal lymph node metastases in patients with esophageal squamous cell carcinoma: a phase 2 single-institution study. Radiat Oncol. 2014;9:28.

31. Kosuga T, Shiozaki A, Fujiwara H, Ichikawa D, Okamoto K, Komatsu S, Otsuji E. Treatment outcome and prognosis of patients with lymph node recurrence of thoracic esophageal squamous cell carcinoma after curative resection. World J Surg. 2011;35:798-804.

\section{Publisher's Note}

Springer Nature remains neutral with regard to jurisdictional claims in published maps and institutional affiliations.
Ready to submit your research? Choose BMC and benefit from:

- fast, convenient online submission

- thorough peer review by experienced researchers in your field

- rapid publication on acceptance

- support for research data, including large and complex data types

- gold Open Access which fosters wider collaboration and increased citations

- maximum visibility for your research: over $100 \mathrm{M}$ website views per year

At $\mathrm{BMC}$, research is always in progress.

Learn more biomedcentral.com/submissions 